\title{
NOTE ON SUBDIRECT SUMS OF RINGS
}

\author{
MASAYOSHI NAGATA
}

In my previous paper "On the theory of semi-local rings," " we saw that if a semi-local ring $R$ with maximal ideals $p_{1}, \ldots, p_{h}$ is a subdirect sum of local rings $R_{\left[p_{i}\right]},{ }^{2)}$ then $R$ is the direct sum of $R_{\left[p_{i}\right]}$ (proposition $15,(\operatorname{sir})^{11}$ ) and that a complete semi-local ring is a direct sum of complete local rings (Remark to proposition 5 , (slr)).

The main purpose of the present note is to prove two kinds of generalization (also for non-commutative case) of the first assertion mentioned above (Theorems 2 and 3). We first introduce in $\S 1$ the concept of $n$-rings and then we define the concepts of semi-local rings, local rings and so on; it is proved here that a commutative (semi-) local ring is a (semi-) local ring in the sense of (slr). It is also remarked that the assumption in Proposition 15, (sir), is a necessary and sufficient condition in order that a commutative semi-local ring is a direct sum of local rings. In $\S 2$, we prove our main theorems. In $\$ 3$, we prove a generalization of the second assertion mentioned above for noncommutative case; in $\S 4$ we study rings which are subdirect sums of (a finite number of) $n$-rings.

\section{Definitions and remarks to commutative case}

Definition 1. A ring ${ }^{31} R$ is called an $n$-ring if $R^{2}=R$ and if for any proper ideal $^{4)} \mathfrak{a}$ in $R$ there exists a maximal ideal ${ }^{51}$ containing $a$.

Definition 2. A quasi-semi-local ring is a non-zero $n$-ring which contains only a finite number of maximal ideals. A quasi-local ring is a non-zero $n$-ring which contains only one maximal ideal.

Definition 3. A quasi-semi-local ring $R$ with maximal ideals $p_{1}, \ldots$, $p_{h}$ is called a semi-local ring if $\bigcap_{i, n} p_{i}^{n}=(0)$. In this case we introduce a topology in $R$ by taking $\left\{\bigcap_{i=1}^{h} \mathfrak{p}_{i}^{n} ; n \stackrel{i, n}{=} 1, \ldots, k, \ldots\right\}$ as a system of neighbour-

Received Sept. 4, 1950.

1) To appear in Proc. Jap. Acad. and will be referred as (slr) in the present note.

2) This notation is same as in (slr); this denotes the topological quotients ring of $p_{i}$ with respect to $R$ : See Chapter I, (sir).

3) A ring means an associative ring.

4) An ideal means a two-sided ideal.

5) Since $R^{2}=R$, any maximal ideal is prime (we say an ideal $p$ in a ring $R$ is maximal if $R \neq \mathfrak{p}$ and if there exists no ideal $\mathfrak{a}$ such as $R \supset \mathfrak{a} \supset \mathfrak{p}$ ). 
hoods of zero; thus a semi-local ring is a topological ring. A local ring is a semi-local, quasi-local ring.

Lemma 1. Let $R$ be a ring and $p_{1}, \ldots, p_{h}$ be proper prime ideals in $R$. Then $\bigcup_{i=1}^{h} p_{i} \neq R^{6}{ }^{6}$

Proof. For $h=1$, our assertion is trivial. So, we assume that $\bigcup_{i=1}^{h-1} p_{i} \neq R$. Let $a$ be an element of $R$ which is not contained in $\bigcup_{i=1}^{h-1} p_{i}$. If $a \notin p_{h}$, our assertion is true; if not, we take an element $b$ of $R$ such as $b \in \bigcap_{i=1}^{h-1} p_{i}, b \notin p_{h},{ }^{*)}$ then $a+b \notin p_{i}$ for any $i(1 \leqq i \leqq h)$. This proves our assertion.

Corollary. Let $R$ be an $n$-ring. Then any union of a finite number of proper ideals does not coincide with $R$.

Proposition 1. A commutative quasi-semi-local ring contains the identity.

Proof. This follows from our Lemma 1 (or Corollary to it) and the fact that a commutative ring $R \neq(0)$ contains the identity if (and only if) there exists an element $a$ of $R$ such that $a R=R$.

Corollary. A commutative semi-local ring is a semi-local ring in the sense of (slr).

We mention, by the way,

Proposition 2. Let a commutative ring $R$ which contains the identity be a direct sum of rings $R_{i}(i=1, \ldots, n)\left(R_{i} \neq(0)\right)$. Let $\left\{\mathfrak{p}_{i \lambda} ; \lambda \in \Lambda_{i}\right\}$ (for each $i=1, \ldots, n)$ be the totality of maximal ideals whose images in $R_{i}$ are different from $R_{i}$. Then $R_{i}$ is the ring of quotients of $S_{i}$ with respect to $R$, where $S_{i}$ is the complementary set of $\bigcup_{\lambda \in \Delta_{i}} p_{i \lambda}$ with respect to $R$. If $R$ is a semi-local ring (or more generally, generalized semi-local ring in the sense of $(\mathrm{s} l \mathrm{r})$ ) then $\boldsymbol{R}_{i}$ coincides also with the topological quotients ring of $S_{i}$ with respect to $R$.

Proof. Easy.

\section{Main theorems}

Lemma 2. Let a ring $R$ be a subdirect sum of rings $R_{i}(i=1, \ldots, n)$. If $\mathfrak{p}$ is a proper prime ideal in $R$, then for at least one $i$ the image of $\mathfrak{p}$ in $R_{i}$ does not coincide with $R_{i}$.

Proof. Let $\mathfrak{n}_{i}$ be the kernel of natural homomorphism of $R$ onto $R_{i}$, for each $i$. Then $\bigcap_{i=1}^{n} \mathfrak{n}_{i}=(0)$. Therefore $\mathfrak{n}_{i} \cong \mathfrak{p}$ for at least one $i$.

Corollary. Let an $n$-ring $R$ be a subdirect sum of rings (necessarily $n$ rings) $R_{i}(i=1, \ldots, n)$. If $\mathfrak{a}$ is a proper ideal in $R$, then for at least one $i$ the

6) Set theoretical union.

*) We may assume without loss of generality that $p_{i} \neq \mathfrak{p}_{j}(i \neq j)$. 
image of $a$ in $R_{i}$ is different from $R_{i}$.

THEOREM 1. Let a ring $R$ be a subdirect sum of $n$-rings $R_{i}(i=1, \ldots, n$ ) $(n>1)$. Then $R$ contains $R_{i}$ if (and only if) the following condition is satisfied: If $\bar{p}_{1}$ and $\bar{p}_{2}$ are two maximal ideals in the direct $\operatorname{sum} \bar{R}$ of $R_{i}(i=1, \ldots, n)$ such that $\bar{p}_{1} \supseteqq R_{1}, \bar{p}_{2}$ 本 $R_{1}$, then $\bar{p}_{1} \cap R \neq \bar{p}_{2} \cap R$ 。

Proof. We set $R_{1} \cap R=a$. We assume that $a \neq R_{1}$. Let $p_{1}$ be a maximal ideal in $R_{1}$ cntaining $a$. Then $p=R \cap\left(p_{1}+R_{2}+\ldots+R_{n}\right)$ is a maximal prime ideal in $R$. On the other hand, $R / a$ is a subdirect sum of rings $R_{i}$ ( $i=2$, $\ldots, n)$. Therefore, for a suitable $k(k>1)$, the image of $\mathfrak{p}$ in $R_{k}$ is different from $R_{k}$ : Let $p k$ be a maximal ideal in $R_{k}$ containing the image of $p$ in $R_{k}$. Then $p$ is contained in $R_{1}+\ldots+R_{k-1}+p_{k}+R_{k+1}+\ldots+R_{n}$. This shows that $R \cap\left(p_{1}+R_{2}+\ldots+R_{n}\right)=R \cap\left(R_{1}+\ldots+R_{k-1}+\mathrm{p}_{k}+R_{k+1}+\ldots+R_{n}\right)$, contrary to our assumption.

Theorem 2. Let a ring $R$ be a subdireci sum of $n$-rings $R_{1}, \ldots, R_{n}$. Then $R$ is the direct sum of $R_{i}(i=1, \ldots, n)$ if (and only if) the following condition is satisfied: If $\bar{p}_{1}$ and $\bar{p}_{2}$ are distinct maximal ideals in the direct sum $\bar{R}$ of $R_{i}$ $(i=1, \ldots, n)$, then $\bar{p}_{1} \cap R \neq \bar{p}_{2} \cap R$.

Proof. This is an immediate consequence of Theorem 1.

COROLIARY 1. If a ring $R$, is a subdirect sum of (quasi-) serni-local rings $R_{1}, \ldots, R_{n}$ and if the number of maximal prime ideals ${ }^{7)}$ of $R$ is the sum of those of $R_{i}$, then $R$ is the direct sum of $R_{i}(i=1, \ldots, n)$.

Corollary 2, A semi-local ring $R$ with maximal ideals $p_{1}, \ldots, p_{h}$ is a direct sum of local rings if and only if each $p_{i}$ is the unique maximal ideal containing $\bigcap_{n=1}^{\infty} p_{i}{ }^{n}$.

Corollary 3. Let a ring $R$ be a subdirect sum of $n$-rings $R_{1}, \ldots, R_{n}$. If $R_{i} / p_{i}$ and $R_{j} / p_{j}$ are non-isomorphic to each other for any maximal ideals $\mathfrak{p}_{i}$ in $R_{i}$ and $n_{j}$ in $R_{j}(i \neq j)$, then $R$ is the direct sum of $R_{1}, \ldots, R_{n}$.

Theorem 3. If an n-ring is a subdirect sum of (quasi-) local rings $R_{i}(i=1$, $\ldots, n)$, then $R$ is a direct sum of suitable $m(\leqq n)$ (quasi-) local rings. (If moreover $R$ contains $n$ distinct maximal ideals, $R$ is the direct sum of $R_{i}$.)

Proof. Our assertion is trivial for the case $n=1$. Now, assuming that our assertion is true for the case $n<h$, we prove the case $n=h$. Let $\bar{R}$ be the direct sum of rings $R_{1}, \ldots, R_{h}$. We set $a_{i}=R \cap R_{i}$. Then $R / a_{i}$ is a subdirect sum of $R_{1}, \ldots, R_{i-1}, R_{i+1}, \ldots, R_{i t}$. Hence $R / a_{i}$ is a direct sum of $m_{i}(<h\rangle$

i) Evidently this number is finite. 
(quasi-) local rings. If $m_{i}<h-1$ for some $i$, our assertion is true because $R$ is a subdirect sum of $m_{i}+1$ (quasi-) local rings. Therefore we assume that $R / \mathfrak{a}_{i} \cong R_{1}+\ldots+R_{i-1}+R_{i+1}+\ldots+R_{h}$ for any $i$. Whence, if $a_{i}=R_{i}$ for some $i$, our assertion is true, i.e., in this case, $R=\bar{R}$. Now, we assume that $a_{i} \neq R_{i}$ (for at least one, therefore any, $i$ ). Let $\bar{p}_{1}, \ldots, \overline{\mathfrak{p}} h$ be the maximal ideals in $\bar{R}$, where $\bar{p}_{i} \cap R_{i} \neq R_{i}$. Set $\bar{p}_{i} \cap R=\mathfrak{p}_{i}$. Since $R / \mathfrak{a}_{i}$ contains only $h-1$ maximal ideals, one $p_{j}$, say $p_{h}$, coincides with some $p_{k}$, say with $p_{h-i}$. Therefore, if $h=2, R$ is itself a (quasi-) local ring. If $h>2, R$ contains elements $\left(b_{1}, 0, \ldots, 0, a_{h}\right)$ and $\left(b_{2}, 0, \ldots, 0, a_{h-1}, 0\right)$ with suitable $b_{1}, b_{2} \in R_{1}$ and $a_{h} \in R_{h}, a_{h_{-1}} \in R_{h_{-1}}$, such that each $a_{i}$ is not contained in the maximal ideal in $R_{i}$. This is a contradiction to our assumption that $p_{h-1}=p_{h}$.

Remark. If a semi-local ring $R$ is a direct sum of semi-local rings $R_{i}$ $(i=1, \ldots, n), R$ is a product space of $R_{i}$.

\section{Complete ${ }^{\text {8) }}$ semi-local rings}

Lemma 3. Let $R$ be a ring such that $R^{2}=R$. If $\mathfrak{a}, \mathfrak{b}$ and $\mathrm{c}$ are ideals in $R$ such that $\mathfrak{a}+\mathfrak{b}=R$ and $\mathfrak{a}+\mathfrak{c}=R$, then $\mathfrak{a}^{m}+b^{n}=R$ for any integers $m$ and $n$, and $\mathfrak{a}+\mathfrak{b c}=R$ (therefore $\mathfrak{a}+(\mathfrak{b} \cap \mathfrak{c})=R$ ).

Proof. Since $a+\mathfrak{b}^{2} \supseteqq R^{2}=R$, we have $a+b^{2}=R$. This proves our first assertion. The second one follows from $R=R^{2} \cong a+b c$.

THEOREM 4. A complete semi-local ring is a direct sum of complete local rings.

Proof. Let $p_{1}, \ldots, p h$ be the totality of maximal ideals in a complete semilocal ring $R$. We set $\mathfrak{a}_{i}{ }^{(n)}=\bigcap_{j \neq i} \mathfrak{p j}^{n}$. By Lemma $3, \mathfrak{p}_{i}{ }^{n}+\mathfrak{a}_{i}{ }^{(n)}=R$. Let $a$ be an element of $R$. Then we can find an element $a_{i, n}$ of $a_{i}{ }^{(n)}$ such that $a_{i, n} \equiv a$ (mod. $\left.\mathfrak{p}_{i}{ }^{n}\right)$. Then the sequence $\left(a_{i, n}\right)(n=1,2, \ldots)$ is convergent (for each $i$ ). Let $f_{i}(a)$ be its limit. Then $f_{i}(a) \equiv a\left(\bmod . \bigcap_{n=1}^{\infty} p_{i}{ }^{n}\right), f_{i}(a) \in \bigcap_{n=1}^{\infty} a_{i}^{(n)} \cdot{ }^{9)} \quad$ This proves that each $p_{i}$ is the unique maximal ideal containing $\bigcap_{n=1}^{\infty} p_{i}^{n}$, i.e., that $R$ is the direct sum of local rings $R_{i}=R /\left(\bigcap_{n=1}^{\infty} p_{i}{ }^{n}\right)(i=1, \ldots, h)$. Comp'eteness of each $R_{i}$ is evident.

\section{Subdirect sums of $n$-rings}

TheOREM 5. Let a ring $R$ be a subdirect sum of $n$-rings $R_{1}, \ldots, R_{n}$. Then

(i) $R$ is an n-ring if (and only if) $R^{2}=R$, and

(ii) $R^{n}$ is an $n$-ring.

Proof. Let $\mathfrak{n}_{i}$ be the kernel of natural homomorphism of $R$ onto $R_{i}$ (for

8) This means topological completeness.

9) This shows that $\sum_{i=1}^{h} f_{i}(a)=a$ and that $R$ is the direct sum of ideals $\prod_{n=1}^{\infty} a^{(i n)}(i=1, \ldots, h)$. 
each $i)$.

(1) Proof of (i).

Let $\mathfrak{a}$ be an ideal in $R$ such that there exists no maximal ideal containing $a$. Then $\mathfrak{a}+\mathfrak{n}_{i}=R$ for each $i$. Therefore $\mathfrak{a}+\left(\bigcap_{i=1}^{n} \mathfrak{n}_{i}\right)=R$, by Lemma 3, i.e., $\mathfrak{a}=R$.

(2) Proof of (ii).

It is clear that $R^{n}$ is a subdirect sum of $R_{1}, \ldots, R_{h}$. Hence, it is sufficient to prove that $R^{n+1}=R^{n}$, by virtue of (i). Evidently $R^{2}+\mathfrak{n}_{i}=R$ for each $i$. Therefore it is easy to see that $R^{n+1}+\mathfrak{n}_{1} \mathfrak{n}_{2} \ldots \mathfrak{n}_{n} \supseteqq R^{n}$, i.e., $R^{n+1}=R^{n}$.

Example. Let $R$ be a ring such that $R^{2}=(0)(R \neq(0))$. Using the notation $(1, R)^{10)}$ as in my paper "On the theory of radicals in a ring" ${ }^{11)}$ we construct a ring $S=R+(1, R)$ (direct sum). Let $\mathfrak{n}_{1}=R, \mathfrak{n}_{2}=\{a+(0, a) ; a \in R\}$. Then $S$ is a subdirect sum of $n$-rings $S / \mathfrak{n}_{1}$ and $S / \mathfrak{n}_{2}$. On the other hand, $S$ is not an $n$-ring because $S^{2}=(1, R)$.

Mathematical Institute, Nagoya University

10) $(1, R)$ is a typical over-ring of a ring $R$ which contains the identity and in which $R$ is an ideal.

11) To appear in J. Math. Soc. Jap. 